\title{
A Saturating Lumped-Parameter Model for an Interior PM Synchronous Machine
}

\author{
Edward C. Lovelace, Member, IEEE, Thomas M. Jahns, Fellow, IEEE, and Jeffrey H. Lang, Fellow, IEEE
}

\begin{abstract}
This paper presents a lumped-parameter magnetic model for an interior permanent-magnet synchronous machine. The model accounts for the effects of saturation through a nonlinear reluctance-element network used to estimate the $q$-axis inductance. The magnetic model is used to calculate inductance and torque in the presence of saturation. Furthermore, these calculations are compared to those from finite-element analysis with good agreement.
\end{abstract}

Index Terms-Generator, interior permanent-magnet synchronous machine, lumped-parameter model, magnetic circuit analysis, magnetic saturation, optimization, starter.

\section{INTRODUCTION}

$\mathbf{T}$ HIS PAPER studies the modeling and design of a saturating interior permanent-magnet (IPM) synchronous machine. Previous lumped-parameter modeling research concerning IPM machines developed and utilized analytical methods for calculating the $d$ - and $q$-axes inductances for the IPM salient rotor structure focussing primarily on unsaturated models with discussions of saturation effects [1]-[4]. This paper presents an explicit nonlinear lumped-parameter model that incorporates the effects of core saturation along the $q$ axis. It is assumed that the $d$ axis is largely unsaturated except at the structural rotor bridges where it is fully saturated at full load; the bridges join the main sections of the rotor core at the air gap to form a unitary lamination. Furthermore, magnetic cross coupling between the $d$ - and $q$-axes is assumed to be negligible [3], [5]. The model is flexible enough to consider some structural design variations while remaining sufficiently tractable for rapid, repetitive design optimizations. The design methodology is applicable, and used, to evaluate designs of various pole numbers and stator slotting distributions. While the analysis focuses on machines with two buried magnets, it can be extended to more magnets.

Paper IPCSD 01-095, presented at the 1999 IEEE International Electric Machines and Drives Conference, Seattle, WA, May 9-12, and approved for publication in the IEEE TRANSACTIONS ON INDUSTRY APPLICATIONS by the Electric Machines Committee of the IEEE Industry Applications Society. Manuscript submitted for review July 1, 1999 and released for publication February 5, 2002. This work was supported by the MIT Consortium on Advanced Automotive Electrical/Electronic Components and Systems.

E. C. Lovelace is with SatCon Technology Corporation, Cambridge, MA 02142 USA (e-mail: ed.lovelace@satcon.com).

T. M. Jahns is with the Wisconsin Electric Machines and Power Electronics Consortium, Department of Electrical and Computer Engineering, University of Wisconsin, Madison, WI 53706-1691 USA (e-mail: jahns@engr.wisc.edu)

J. H. Lang is with the Laboratory for Electromagnetic and Electronic Systems, Department of Electrical Engineering and Computer Science, Massachusetts Institute of Technology, Cambridge, MA 02139 USA (e-mail: lang@mit.edu)

Publisher Item Identifier S 0093-9994(02)04515-2.

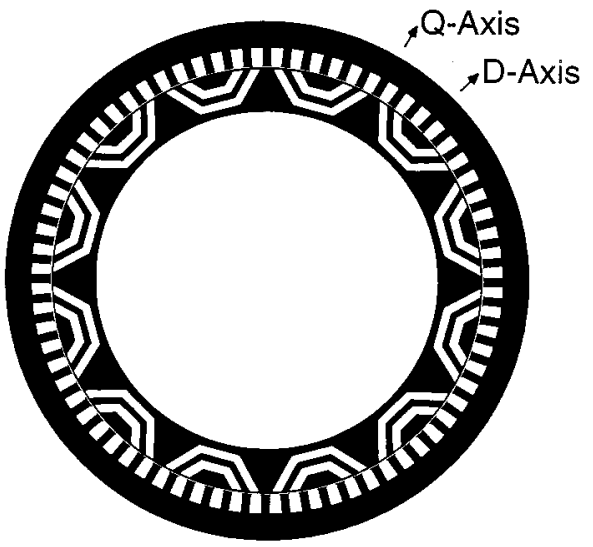

Fig. 1. Cross section of a 12-pole IPM machine.

Fig. 1 shows a cross section of a 12-pole IPM machine. In this configuration, each rotor pole contains two buried magnets that are magnetized across their shorter dimensions along the $d$ axis. The stator of the IPM machine is excited with polyphase balanced sinusoidal currents to produce the characteristic synchronously rotating magnetomotive force (MMF) wave. Control of torque (motoring) or electrical power output (generating) can be achieved using vector control methods by varying the proportion of the $d$ - and $q$-axes currents. The inherent saliency affords the use of flux weakening along the $d$ axis to obtain a wide constant power speed range (CPSR) [6]-[8]. Additionally, magnet strength and/or volume requirements are typically lower than surface PM machines. These two attributes of IPM machines combine to make them very attractive for spindle and traction applications [4], [9], [10]. An appropriately designed IPM machine for such wide CPSR applications has the potential for good inverter utilization and, therefore, lower semiconductor current ratings and cost. Combined with the limited machine material costs due to the limited use of magnets, an IPM machine drive has the potential for the lowest overall drive system cost in wide CPSR applications. However, as noted above, the magnetic characteristics of IPM machines is such that equivalent magnetic circuits for properly designed machines are nonlinear, hence, the motivation for the study of magnetically saturable IPM machine models.

\section{EleCtromagnetic MODEL}

\section{A. Electromagnetic Torque}

We are primarily interested in calculating the electromagnetic torque produced by the IPM machine through a lumped-parameter analysis. The well-known salient synchronous machine 


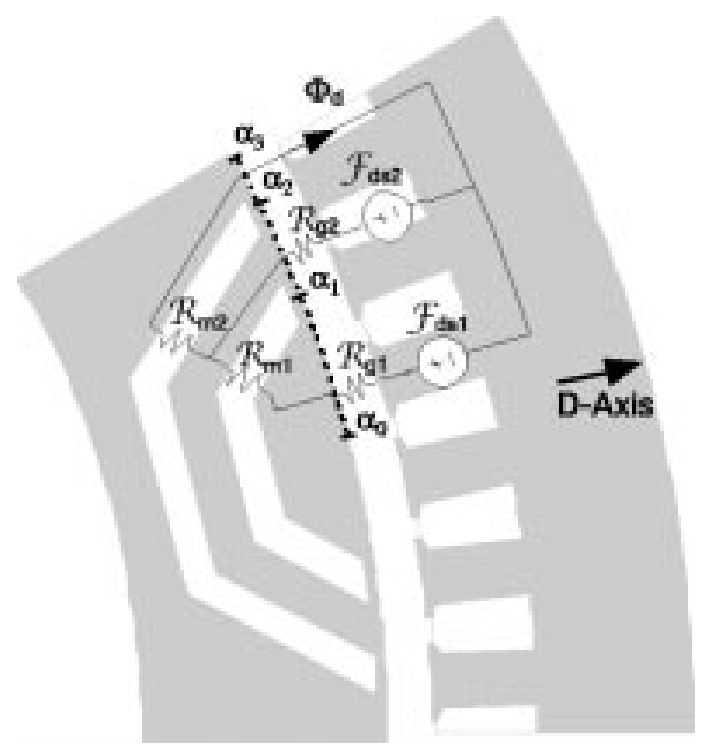

Fig. 2. Single pole of an IPM machine overlaid with an equivalent magnetic circuit to estimate $d$-axis inductance.

equation provides a good estimate for linear analysis [11]. For a three-phase machine, it is

$$
T=3 p\left(\lambda_{\mathrm{PM}}-\left(L_{q}-L_{d}\right) I_{d}\right) I_{q}
$$

where $p$ is the number of pole pairs, $\lambda_{\mathrm{PM}}$ is the fundamental flux linkage due to the PMs, and the inductances and currents, $L_{d}, L_{q}, I_{d}$, and $I_{q}$, are defined such that the $d$ axis is coincident with the fundamental PM flux axis as shown in Fig. 1. Although (1) is typically applied to lumped-parameter analysis of machines using linear magnetic materials, the formula structure is still applicable if the inductances are implicit functions of the excitation when modeling real materials that saturate magnetically.

\section{B. d-Axis Inductance}

The $d$ - and $q$-axes inductances are estimated using equivalent magnet circuits. First, the $d$-axis inductance is determined from inductance ratios defined with respect to an unsaturated $q$-axis inductance model. Then, the saturated $q$-axis inductance is calculated from a nonlinear magnetic circuit model.

For the given rotor cross section, the unsaturated $q$-axis magnetizing inductance $L_{q m}$ can be calculated from the round-rotor air-gap inductance $L_{\mathrm{ag}}$ according to

$$
L_{q m}=L_{\mathrm{ag}} .
$$

The $q$-axis inductance is then just the sum of the magnetizing component and the leakage term

$$
L_{q}=L_{q m}+L_{l} .
$$

Vagati et al. developed analytical relationships based on magnetic circuit analysis between the unsaturated $q$-axis inductance to the $d$-axis inductance [1], [2]. That $d$-axis model is summarized as follows and its equations are applied without derivation. For the rotor cross section from Fig. 1, the equivalent $d$-axis magnetic circuit is chosen as shown Fig. 2, where $\mathcal{R}_{m k}, \mathcal{R}_{g k}$, and $\mathcal{F}_{d s k}$ are the magnet reluctance, air-gap reluctance, and $d$-axis stator MMF, respectively, from the $k$ th section of the machine pole cross section.

The ratio between the $q$-axis magnetizing inductance and the "through" $d$-axis inductance component $L_{d t}$ is then given by

$$
\frac{L_{d t}}{L_{q m}}=\frac{4}{\pi} \sum_{k} f_{d s k}\left(f_{d s k}-f_{d r k}\right)\left(\alpha_{k}-\alpha_{k-1}\right)
$$

where $k=1,2$ and $\alpha_{k}-\alpha_{k-1}$ is the angular span across each path at the air gap [1], [2]. The per-unit quantities for the air-gap MMF on the stator side $f_{d s k}$ are defined because they are convenient for expressing the dimensionless inductance ratio. To express the ratio, it is also necessary to solve the linear circuit for the potentials, $f_{d r k}$, on the rotor side of the air gap. $L_{d t}$ represents the flux that fully links the magnet cavities. By contrast, the "circulating" $d$-axis inductance component $L_{d c}$ represents the flux that only crosses the air gap and the structural rotor bridges. $L_{d c}$ is given by [1], [2]

$$
\frac{L_{d c}}{L_{q m}}=1-\frac{4}{\pi} \sum_{k}\left(\alpha_{k}-\alpha_{k-1}\right) f_{d s k}^{2} .
$$

The ratios for $L_{d t}$ and $L_{d c}$ are summed to obtain the $d$-axis magnetizing inductance

$$
L_{d m}=\left(\left(\frac{L_{d t}}{L_{q m}}\right)+\left(\frac{L_{d c}}{L_{q m}}\right)\right) L_{q m} .
$$

Similarly, the total $d$-axis inductance is then

$$
L_{d}=L_{d m}+L_{l} .
$$

\section{C. q-Axis Inductance}

High-performance operation often bulk saturates the $q$ axis, which makes the infinitely permeable steel assumption implicit in $L_{q m}$ inappropriate for estimating $L_{q}$ [2]. The magnet cavities provide a flux barrier on the $d$ axis producing an inverse saliency $L_{q}>L_{d}$. This makes it reasonable to assume that the $q$ axis will saturate first, and furthermore, that performance will be degraded due to lower saliency as the $d$ axis starts to saturate beyond the structural rotor bridges. For this analysis, $L_{q m}$ is replaced by a nonlinear function of $I_{q}$ defined as the saturable $q$-axis magnetizing inductance

$$
L_{q m s}=L_{q m s}\left(I_{q}\right) .
$$

The total $q$-axis inductance including saturation effects then replaces (3) and is given by

$$
L_{q}=L_{q m s}+L_{l} .
$$

Equations (1), (2), and (4)-(9) form an electrical torque model that accounts for the effects of magnetic saturation. Note that the unsaturated $q$-axis magnetizing inductance $L_{q m}$ is still appropriate for calculating the $d$-axis inductance as in (4)-(7) since the $d$ axis does not bulk saturate.

Finite-element analyses (FEAs) are often conducted to calculate the $q$-axis inductance or to produce scaling factors to apply to an unsaturated inductance equation [12], [13]. By contrast, an equivalent magnetic circuit model is employed here for design flexibility and solution speed as discussed in Section II-B. 


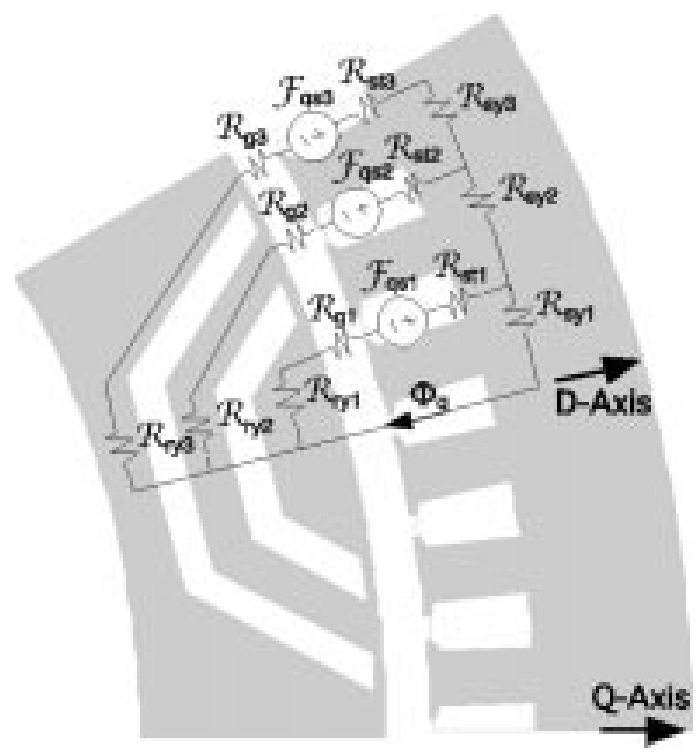

Fig. 3. Single pole of an IPM machine overlaid with an equivalent magnetic circuit to estimate the saturating $q$-axis inductance.

The following paragraphs describe the method used to estimate the saturated $q$-axis inductance from the 15-element equivalent magnetic circuit shown in Fig. 3. There are three rotor yoke reluctances $\mathcal{R}_{\text {ry1 }}, \mathcal{R}_{\text {ry2}}$, and $\mathcal{R}_{\text {ry3 }}$ which model the core flux paths. The flux through each of these paths is assumed to flow separately through the three corresponding linear air-gap reluctances $\mathcal{R}_{g 1}, \mathcal{R}_{g 2}$, and $\mathcal{R}_{g 3}$. Similarly, the stator coil excitation and tooth reluctances are modeled by the three corresponding parallel paths consisting of an MMF source $\mathcal{F}_{q s 1}, \mathcal{F}_{q s 2}$, or $\mathcal{F}_{q s 3}$ and series reluctance $\mathcal{R}_{s t 1}, \mathcal{R}_{s t 2}$, or $\mathcal{R}_{s t 3}$. Finally, the stator back iron is modeled by three more reluctances $\mathcal{R}_{s y 1}, \mathcal{R}_{s y 2}$, and $\mathcal{R}_{\text {sy } 3 \text {. }}$

Each nonlinear reluctance representing the segments of the stator and rotor core $\mathcal{R}_{i}$ is defined by

$$
\mathcal{R}_{i}=\frac{\mathcal{F}_{i}}{\Phi_{i}\left(\mathcal{F}_{i}\right)}=\frac{l_{i}}{\mu\left(B_{i}\right) A_{i}}
$$

where $\mathcal{F}_{i}$ is the MMF drop across, and $\Phi_{i}\left(\mathcal{F}_{i}\right)$ is the flux through the $i$ th section of the core. The linear air-gap reluctance elements are given by

$$
\mathcal{R}_{g k}=\frac{g^{\prime}}{\mu_{o}\left(\alpha_{k}-\alpha_{k-1}\right) r_{\mathrm{ag}} l}
$$

where $r_{\text {ag }}$ is the air-gap radius, $l$ the active machine length, $g^{\prime}$ is the equivalent air gap that accounts for the permeance effects of the stator slotting [11], $k=1,2,3$, and $\alpha_{k}-\alpha_{k-1}$ is the angular span across each path at the air gap.

As shown in (10), the $i$ th section reluctance $\mathcal{R}_{i}$ can be estimated from an equivalent cross-sectional area $A_{i}$ and length $l_{i}$ for each section represented by the magnetic circuit. Fig. 4 shows the approximate line lengths and per-unit depth areas for the example IPM machine cross section of Fig. 1. The nonlinear core material magnetization characteristic is given by a tabular permeability function $\mu\left(B_{i}\right)$.

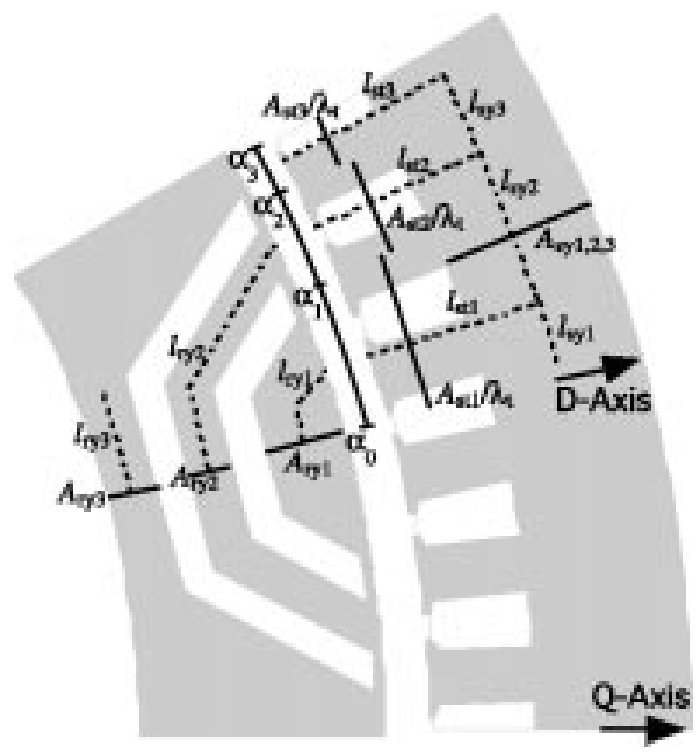

Fig. 4. Sketch of $A_{i}$ (per-unit depth) and $l_{i}$ for calculating the nonlinear reluctance elements, $\mathcal{R}_{i}$.

The three stator MMF source elements $\mathcal{F}_{q s k}$ are calculated from the average MMF across each path produced by the equivalent sinusoidal stator MMF wave with fundamental rms amplitude $\mathcal{F}_{s 1}$

$$
\mathcal{F}_{q s k}=\frac{1}{\alpha_{k}-\alpha_{k-1}} \int_{\alpha_{k-1}}^{\alpha_{k}}\left(\mathcal{F}_{s 1} \sin \alpha\right) d \alpha
$$

This model neglects stator saliency.

Careful selection of the $A_{i}$ and $l_{i}$ values is necessary to produce a reasonably accurate $q$-axis inductance prediction. The geometry is such that the physical value of these parameters can change value significantly from point to point along any particular flux path in the $i$ th section represented by $\mathcal{R}_{i}$. This is particularly true for the rotor yoke elements. Using a combination of reasonable limits on the magnet cavity geometries the $A_{i}$ and $l_{i}$ values can be approximated by average values in each section of the rotor. Such limits include forcing the narrowest cross-sectional area on the "ry3" rotor yoke to be on the $d$-axis symmetry line, and forcing the two magnet cavities to be near to parallel. $\mathcal{R}_{\text {ry3 }}$, in particular, is estimated by ignoring the beginning and ending thirds of the path and only calculating $A_{\text {ry3 }}$ and $l_{\mathrm{ry} 3}$ where the cross-sectional area is smallest between the magnet and the rotor inner radius. The particular orientation of the stator teeth with respect to the rotor features is ignored. The flux entering the teeth is treated by scaling with respect to the slot pitch fraction $\lambda_{t}$ as indicated in Fig. 4.

For a given stator excitation current, the nonlinear network equations are solved iteratively for the unknown $\Phi_{i}$ and $\mathcal{F}_{i}$. A piecewise-constant air-gap flux can then be constructed from the three parallel rotor flux solutions $\Phi_{\mathrm{ry} 1}, \Phi_{\mathrm{ry} 2}$, and $\Phi_{\mathrm{ry} 3}$. The $q$-axis inductance is calculated by Fourier analysis of the piecewise-constant air-gap flux, the given current excitation, and the known winding parameters.

This methodology is further illustrated in the following figures. Table I shows the machine specifications that were analyzed and that match the geometry shown in Fig. 1. 
TABLE I

IPM MACHINE SPECIFICATIONS

\begin{tabular}{l|l}
\hline Poles & 12 \\
\hline Active Length & $58 \mathrm{~mm}$ \\
\hline Air Gap Diameter & $246 \mathrm{~mm}$ \\
\hline Air Gap & $0.635 \mathrm{~mm}$ \\
\hline Rated Current & $273 \mathrm{~A} \mathrm{RMS}$ \\
\hline Nominal PM Remanence & $0.21 \mathrm{~T}$ \\
\hline
\end{tabular}

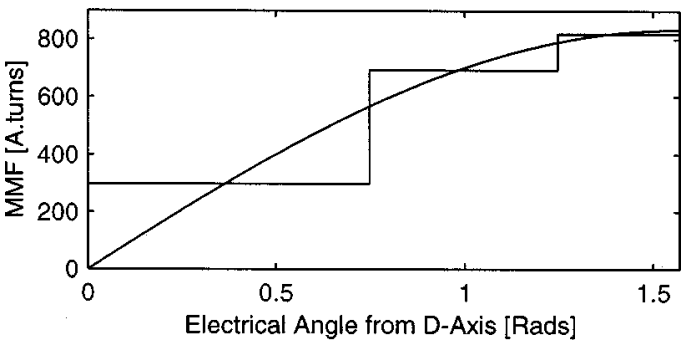

(a)

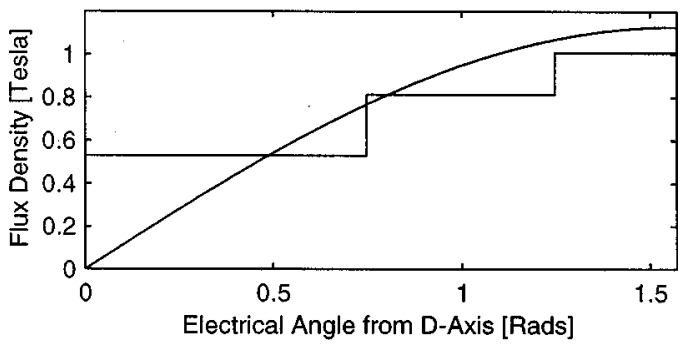

(b)

Fig. 5. (a) Fundamental and magnetic circuit element MMF stator excitation. (b) Resulting magnetic circuit element air-gap flux density and associated fundamental air-gap flux density.

Fig. 5(a) shows the fundamental stator source MMF wave over a half-pole section and its decomposition into the three MMF source elements $\mathcal{F}_{q s k}$ for a given current excitation. Fig. 5(b) shows solutions for the air-gap flux $\Phi_{g k}$ in each parallel path of the nonlinear model for the given excitation. The Fourier-calculated fundamental flux is also shown. Fig. 6 shows the relative degree of saturation in each of the parallel rotor circuit elements as the excitation is increased.

It is worth noting here that the information conveyed by Fig. 6 illustrates the power of the lumped-parameter modeling beyond just speed of design analysis. From such views, design modifications can be made with some intelligence. For example, since the onset of magnetic saturation for a high-performance electrical steel (M19 is used in this analysis) occurs in the range $1.8-2.1 \mathrm{~T}$ it is observed that the "ry2" section is heavily saturated while the other rotor sections are not. Although the "ry1" and "ry3" sections appear to magnetically saturate, the flux is simply being limited by the relative reluctance of the different magnetic circuit paths in the nonlinear network. As such, there may be some material utilization benefit to shrinking the "ry1" and "ry3" sections and increasing the cross-sectional size of the "ry2" section.

\section{PM Flux}

The PM flux linkage parameter $\lambda_{\mathrm{PM}}$ is calculated from a linear magnetic circuit model similar to that used to estimate

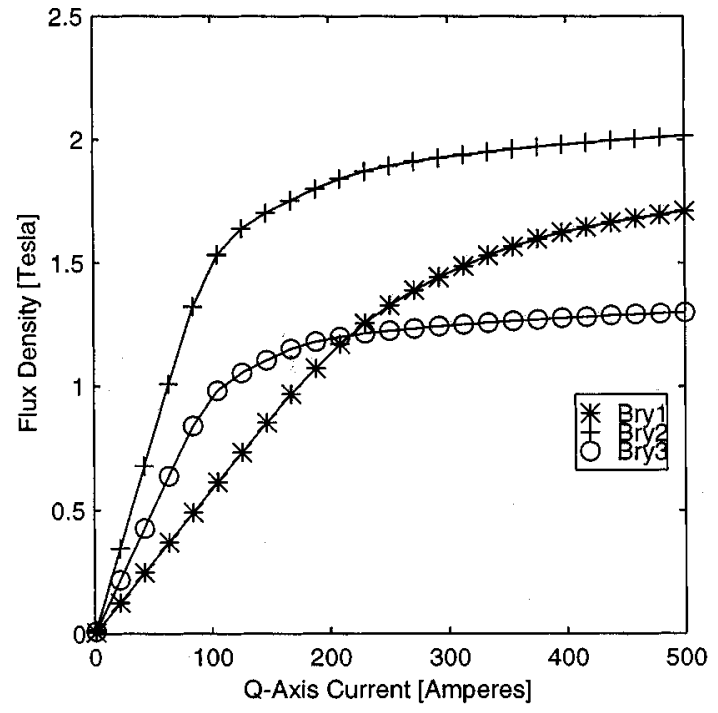

Fig. 6. Average flux density in each rotor yoke reluctance element as the stator excitation is increased.

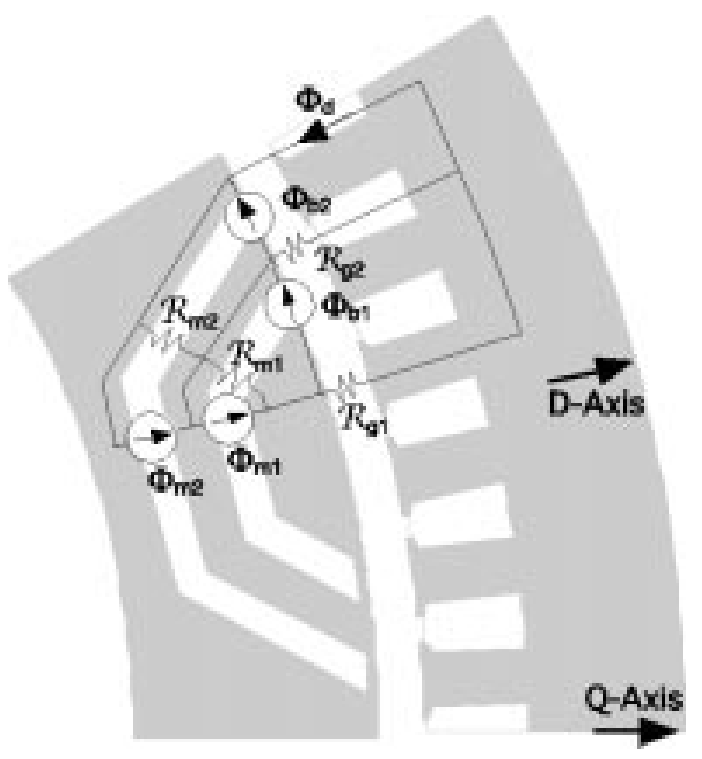

Fig. 7. Linear circuit model used to calculate the fundamental permanent magnet flux produced on the $d$ axis.

$L_{d}$ [1]. Fig. 7 shows the corresponding equivalent circuit model where the PM layers are modeled as flux sources with parallel reluctance, and the structural rotor bridges are represented by saturated flux sources. The PM sources are given by the magnet remanence $B_{r}$. The rotor bridge sources are in the direction that reduces the overall air-gap flux acting as a leakage path within the rotor. It is assumed that the bridges saturate to an effective constant value $B_{s}$.

The magnet layer reluctance elements are given by

$$
\mathcal{R}_{m k}=\frac{h_{m k}}{\mu_{0} A_{m k}}
$$

where the cross-sectional areas are

$$
A_{m k}=l_{m k} l
$$




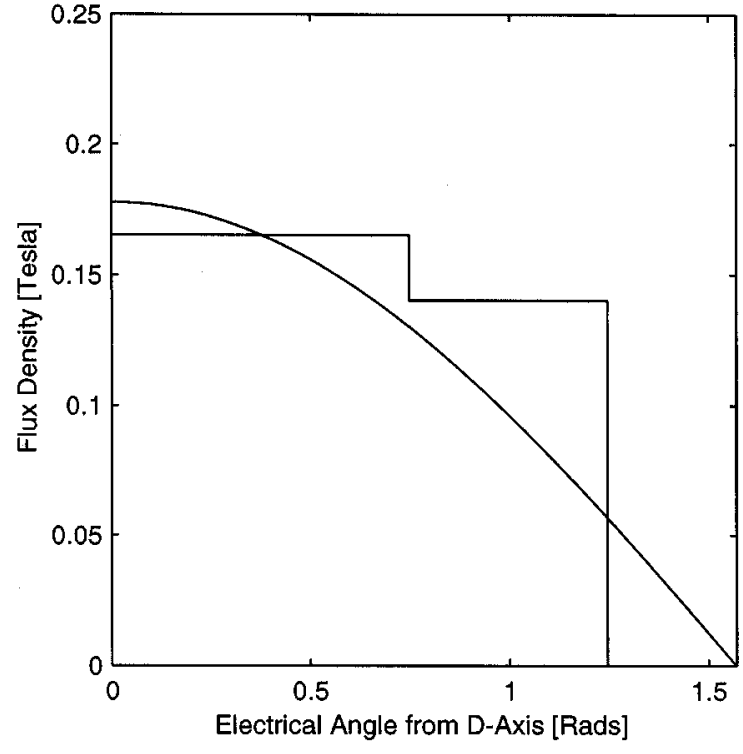

Fig. 8. Permanent-magnet air-gap flux density calculated from the $\lambda_{\mathrm{PM}}$ linear magnetic circuit model and the resulting fundamental component.

where $l_{m k}$ is the average length from tip to tip, $h_{m k}$ is the thickness of the $k$ th magnet layer, and $k=1,2$. The cross-sectional areas of the bridges are

$$
A_{b k}=h_{b k} l
$$

where $h_{b k}$ is the thickness of the $k$ th bridge.

After analyzing the linear system for the flux through each of the air-gap circuit branches and rearranging terms, the flux density in each branch can be written as

$$
B_{g k}=\frac{A_{r e q k} B_{r}+A_{s e q k} B_{s}}{A_{g k}}
$$

where

$$
\begin{aligned}
& A_{\text {req } 1}=\frac{h_{m 2} \mathcal{R}_{g 2}+h_{m 1}\left(\mathcal{R}_{g 1}+\mathcal{R}_{g 2}\right)}{\mu_{0}\left(\mathcal{R}_{m 2} \mathcal{R}_{g 2}+\left(\mathcal{R}_{m 2}+\mathcal{R}_{g 2}\right)\left(\mathcal{R}_{m 1}+\mathcal{R}_{g 1}\right)\right)} \\
& A_{\text {req2 }}=\frac{h_{m 2}\left(\mathcal{R}_{m 1}+\mathcal{R}_{g 1}\right)-h_{m 1} \mathcal{R}_{m 2}}{\mu_{0}\left(\mathcal{R}_{m 2} \mathcal{R}_{g 2}+\left(\mathcal{R}_{m 2}+\mathcal{R}_{g 2}\right)\left(\mathcal{R}_{m 1}+\mathcal{R}_{g 1}\right)\right)} \\
& A_{s e q 1}=-\frac{\mathcal{R}_{m 1}\left(\mathcal{R}_{m 2}+\mathcal{R}_{g 2}\right) A_{b 1}+\mathcal{R}_{m 2} \mathcal{R}_{g 2} A_{b 2}}{\mathcal{R}_{m 2} \mathcal{R}_{g 2}+\left(\mathcal{R}_{m 1}+\mathcal{R}_{g 1}\right)\left(\mathcal{R}_{m 2}+\mathcal{R}_{g 2}\right)} \\
& A_{\text {seq2 }}=\frac{\mathcal{R}_{m 1} \mathcal{R}_{m 2} A_{b 1}-\mathcal{R}_{m 2}\left(\mathcal{R}_{m 1}+\mathcal{R}_{g 1}\right) A_{b 2}}{\mathcal{R}_{m 2} \mathcal{R}_{g 2}+\left(\mathcal{R}_{m 1}+\mathcal{R}_{g 1}\right)\left(\mathcal{R}_{m 2}+\mathcal{R}_{g 2}\right)}
\end{aligned}
$$

$\lambda_{\mathrm{PM}}$ is then determined by Fourier analysis of the piecewiseconstant air-gap flux estimate and the known stator winding constants. Fig. 8 shows the resultant air-gap flux in each element. The calculated fundamental air-gap flux density is overlaid.

\section{INDUCTANCE AND TORQUE PREDICTIONS}

To validate the model, the predictions are compared to FEA estimates for a candidate IPM machine design. A comparison of the inductances and electromagnetic torque estimates from

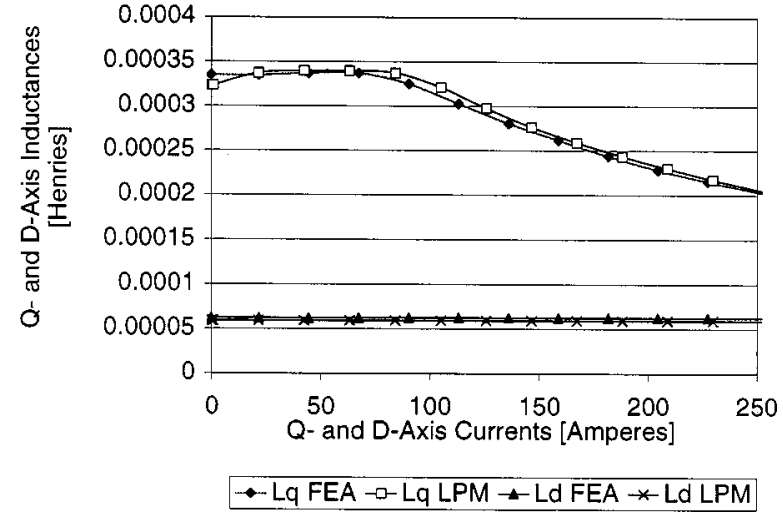

Fig. 9. Comparison of $q^{-}$and $d$-axes inductance calculations by FEA and lumped-parameter models (LPM).

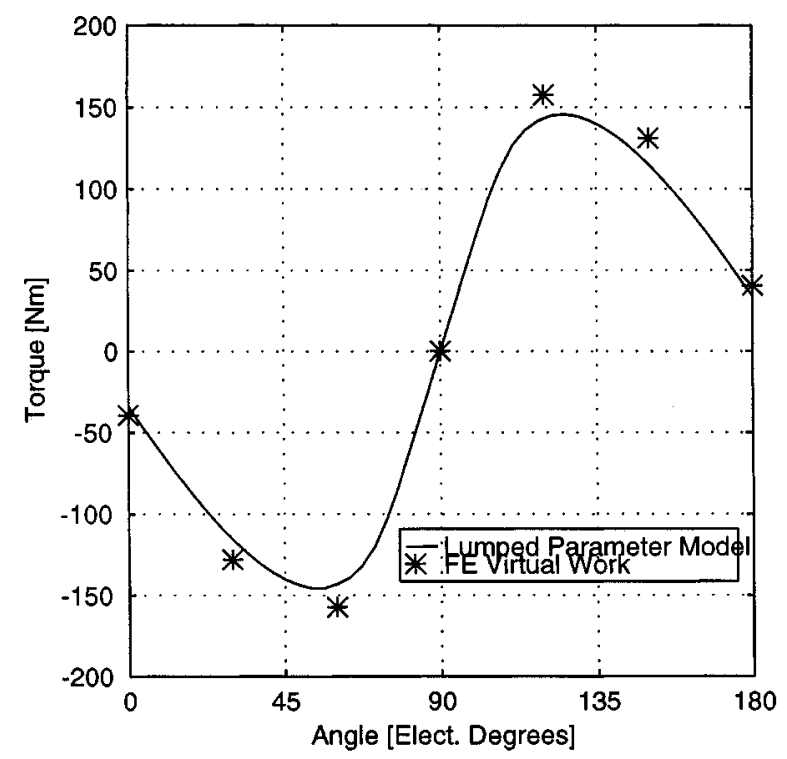

Fig. 10. Comparison of electromagnetic torque calculation by FEA and lumped-parameter model versus torque angle with constant rms phase current.

FEA and the lumped-parameter model are shown in Figs. 9 and 10. Ansoft's Maxwell 2D software was employed for the FEA results. Although only one example is shown, similar results have been achieved for a number of designs that have two PM layers in the rotor.

Both the $q$-axis and $d$-axis inductance calculations shown in Fig. 9 agree nearly perfectly for the two methods: FEA and lumped-parameter modeling. The FEA $d$ - and $q$-axes inductances were calculated from the known winding characteristics and the stored energy in the mesh solutions for stator excitation on the $d$ - and $q$-axes, respectively. Note the inflection in the $q$-axis inductance curve for very low currents. This corresponds to an inflection in the BH curve for the material, M19 steel for this case. For the range of current shown in the figure the total saliency ratio goes from a peak of 5.4 down to 3.1 at higher $q$-axis current. Although this is lower than other reported synchronous reluctance saliency ratios (up to a ratio of 8 for this structure), it should be noted that the machine studied here has a higher pole count, and it is analyzed with a saturating model, both of which contribute to a lower overall saliency ratio [1], [2]. 
The FEA electromagnetic torque predictions in Fig. 10 also corroborate the lumped-parameter model calculations. For this result, the rms phase current is held constant, and the torque angle is swept through the range of interest. It should be noted, though, that this result does not show the torque ripple with respect to the slotting effects of the stator. The averaged lumpedparameter model does not capture torque ripple, so for a meaningful comparison the tooth positioning for the FEA was chosen to approximately achieve the average torque produced at each torque angle.

\section{CONCLUSIONS}

The nonlinear lumped-parameter model produces accurate estimates of $q$ - and $d$-axes inductances, and electromagnetic torque as evidenced by the FEA comparisons. The utility of the lumped-parameter method is twofold. The underlying magnetic circuit can be easily analyzed to investigate characteristics of a machine design such as heavy saturation in particular portions of the core. In this research, the primary benefit is that the lumped-parameter model is easily incorporated into design optimization programs that benefit from rapid solution methods [4].

\section{REFERENCES}

[1] A. Fratta, A. Vagati, and F. Villata, "Design criteria of an IPM machine suitable for field-weakened operation," in Proc. ICEM'90, 1990, pp. $1059-1065$

[2] T. A. Lipo, T. J. E. Miller, A. Vagati, I. Boldea, L. Malesani, and T. Fukao, "Synchronous reluctance drives tutorial," presented at the IEEE-IAS Annu. Meeting, 1994.

[3] A. Vagati, A. Fratta, G. Franceschini, and P. M. Rosso, "A.C. motors for high-performance drives: A design-based comparison," in Conf. Rec. IEEE-IAS Annu. Meeting, 1995, pp. 725-733.

[4] E. C. Lovelace, T. M. Jahns, J. L. Kirtley Jr., and J. H. Lang, "An interior PM starter/alternator for automotive applications," in Proc. ICEM'98, vol. 3, Istanbul, Turkey, 1998, pp. 1802-1808.

[5] A. Vagati, M. Pastorelli, and G. Franceschini, "Effect of magnetic cross-coupling in synchronous reluctance motors," Intell. Motion, pp. 279-285, 1997

[6] R. Schiferl and T. A. Lipo, "Power capability of salient pole P.M. synchronous motors in variable speed drive," in Conf. Rec. IEEE-IAS Annu. Meeting, 1988, pp. 23-31.

[7] T. M. Jahns, "Flux weakening regime operation of an IPM synchronous motor drive," IEEE Trans. Ind. Applicat., vol. IA-23, pp. 681-689, July/Aug. 1987.

[8] W. L. Soong and T. J. E. Miller, "Field-weakening performance of brushless synchronous AC motor drives," Proc. IEE-Elect. Power Applicat., vol. 141, pp. 331-339, 1994.

[9] _ - "Theoretical limitations to the field-weakening performance of the five classes of brushless synchronous AC motor drive," in Proc. IEE Electric Machines and Drives Conf., 1993, pp. 127-132.

[10] —, "Practical field-weakening performance of the five classes of brushless synchronous AC motor drive," in Proc. European Power Electronics Assoc. Conf., 1993, pp. 303-310.

[11] T. J. E. Miller, Brushless Permanent-Magnet and Reluctance Motor Drives. Oxford, U.K.: Clarendon, 1989.

[12] W. Soong, "Design and modeling of axially-laminated interior permanent magnet motor drives for field-weakening applications," Ph.D. dissertation, Dept. Electron. Elect. Eng., Univ. Glasgow, Glasgow, U.K., 1993.

[13] I. Boldea, Reluctance Synchronous Machines and Drives. Oxford, U.K.: Clarendon, 1996.

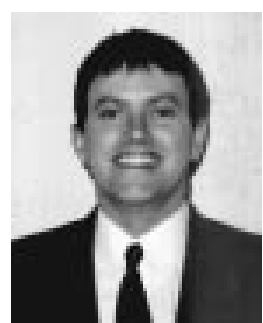

Edward C. Lovelace (S'96-M'00) received the S.B. and S.M. degrees in 1988 and 1996, respectively, from the Department of Mechanical Engineering, and the S.M. and Ph.D. degrees in 1996 and 2000, respectively, from the Department of Electrical Engineering and Computer Science, Massachusetts Institute of Technology (MIT), Cambridge.

In May, 2000, he joined SatCon Technology Corporation, Cambridge, MA, which performs research and development in electric power and energy products, and is currently the Mechanical/Electromagnetics Group Leader. From 1988 to 1996, he was with GE Aircraft Engines, Lynn, MA, where he was involved with development of advanced engine control system designs, military and commercial data communications systems, and ground and flight testing for hydromechanical and digitally controlled engine systems. When he returned to MIT in 1994, he continued to specialize in the design and control of electric machine drives for transportation applications. His current technology research and development interests are mechatronics, advanced machine design, controls, and transportation applications.

Dr. Lovelace received a full three-year fellowship from the Dwight D. Eisenhower Fellowship Program (U.S. Department of Transportation, Federal Highway Administration ISTEA grant and fellowship program) for his doctoral research. Further sponsorship came from the MIT/Industry Automotive Consortium on Advanced Electrical/Electronic Components and Systems with which he was a Research Assistant on automotive electronics research focusing on transition to a 42-Vdc vehicle platform. He was also the recipient of the Technology and Policy Program Cooke Public Service Award and the Best Master Works Oral Thesis Presentation Award.

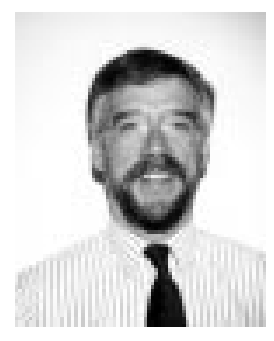

Thomas M. Jahns (S'73-M'79-SM'91-F'93) received the S.B. and S.M. degrees in 1974 and the $\mathrm{Ph} . \mathrm{D}$. degree in 1978 from Massachusetts Institute of Technology, Cambridge, all in electrical engineering.

In 1998, he joined the faculty of the University of Wisconsin, Madison, as a Professor in the Department of Electrical and Computer Engineering, where he is also an Associate Director of the Wisconsin Electric Machines and Power Electronics Consortium (WEMPEC). Prior to joining the University of Wisconsin, he was with GE Corporate Research and Development, Schenectady, NY, for 15 years, where he pursued new power electronics and motor drive technology in a variety of research and management positions. His research interests include permanent-magnet synchronous machines for a variety of applications ranging from high-performance machine tools to low-cost appliance drives. During 1996-1998, he conducted a research sabbatical at Massachusetts Institute of Technology, where he directed research activities in the area of advanced automotive electrical systems and accessories as co-director of an industry-sponsored automotive consortium.

Dr. Jahns was awarded the William E. Newell Award by the IEEE Power Electronics Society (PELS) in 1999. He has been recognized as a Distinguished Lecturer by the IEEE Industry Applications Society (IAS) during 1994-1995 and by PELS during 1998-2000. He has served as President of PELS (1995-1996) and as a Member of the IAS Executive Board between 1992-2001.

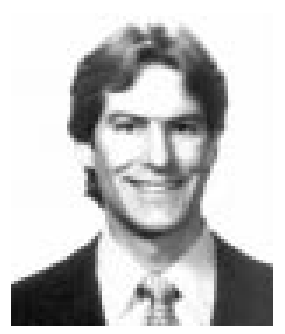

Jeffrey H. Lang (S'78-M'79-SM'95-F'98) received the S.B. and S.M. degrees in electrical engineering and the $\mathrm{Ph} . \mathrm{D}$. degree from Massachusetts Institute of Technology (MIT), Cambridge, in 1975, 1977, and 1980, respectively.

$\mathrm{He}$ is a Professor of Electrical Engineering at MIT and Associate Director of the MIT Laboratory for Electromagnetic and Electronic Systems. He has been an MIT faculty member since 1980 . His research and teaching interests focus on the analysis, design, and control of electromechanical systems with an emphasis on rotating machinery, micro sensors and actuators, and flexible structures. He has authored more than 140 technical papers and is the holder of five patents in the areas of electromechanics, power electronics, and applied control. He is a former Associate Editor of Sensors and Actuators.

Prof. Lang has been awarded three Best Paper Prizes by various IEEE Societies. He is a former Hertz Foundation Fellow. 